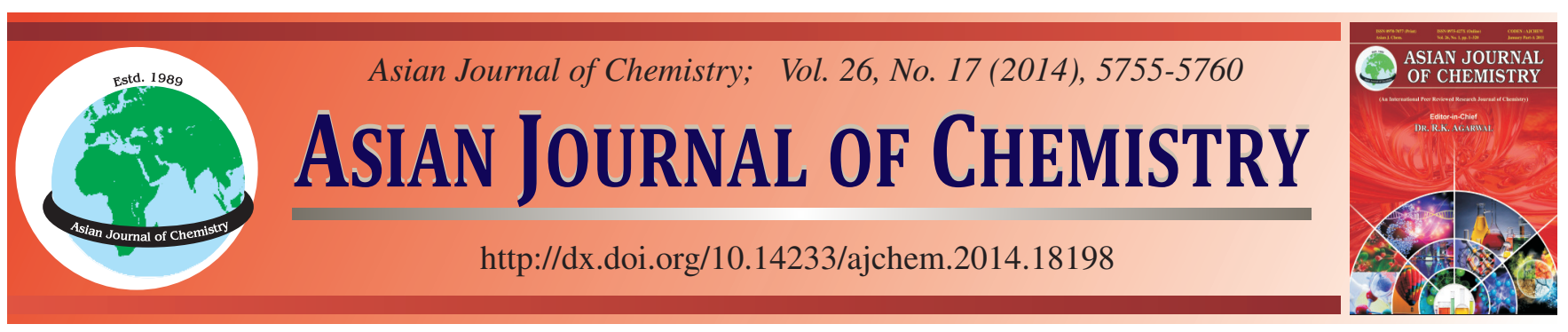

\title{
Effects of Different Macromolecule Absorbent Resins on the Moisture Characteristics of Major Soil Type on Loess Plateau $\dagger$
}

\author{
Yong-Hui YANG $^{1,2,3}, \mathrm{~J}_{\mathrm{I}}$-ChenG $\mathrm{WU}^{1,3}$ and SHI-Wei ZhaO ${ }^{2, *}$
}

${ }^{1}$ Institute of Plant Nutrition, Environment and Resources, Henan Academy of Agricultural Sciences, Zhengzhou 450002, P.R. China

${ }^{2}$ Institute of Soil and Water Conservation, Northwest A \& F University, Yangling 712100, P.R. China

${ }^{3}$ Yuanyang Experimental Station of Crop Water Use, Ministry of Agriculture, Yuanyang 453514, P.R. China

*Corresponding author: E-mail: swzhao@ms.iswc.ac.cn

In order to verify the effects of different macromolecule absorbent resins [PAM (polyacrylamide), PAA (sodium polyacrylate), Wote super absorbent] on the water characteristics of Heilu soil, the major type of soil on the Loess Plateau. We studied the effects of different macromolecule absorbent resins on the soil moisture characteristic curve, water retention ability, water furnishing ability, water transmitting ability and evaporative resistance. The application of different macromolecule absorbent resins into the soil improved the water retention ability and water furnishing ability of the soil and promoted the increase of availability of soil moisture. In addition, it also improved the soil moisture constant and reduced the evaporation capacity of the soil. Among the various applications, the application with $0.212 \mathrm{mg} / \mathrm{kg}$ of sodium polyacrylate was the most ideal choice for improving the water retention and water furnishing abilities and increasing the availability of soil moisture of Heilu soil among various macromolecule absorbent resins.

Keywords: Macromolecular materials, Loess Plateau, Heilu soil, Water retention ability, Water furnishing ability, Available water.

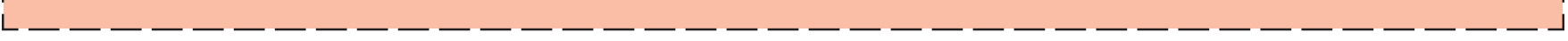

\section{INTRODUCTION}

Macromolecule absorbent resins have an incredibly strong water absorbing capacity and can absorb moisture content several hundred or even thousand times higher than that of its own. More than $85 \%$ of the absorbed moisture may be used by crops. Due to the cross linkage of molecular structure, the moisture absorbed by the molecular network cannot be extracted using general physical methods, thus contributing to the strength of the water retentivity.

Due to its unique structure and excellent performance, PAM (polyacrylamide) has been gradually applied in the restoration and improvement of the ecological environment ${ }^{1,2}$. With its strong water absorption and water retention abilities, PAM can significantly improve the water retention ability and moisture of soil and improve the utilization efficiency of water resources after application in soil as a non-toxic and non-polluting material $^{3}$. As shown by a large number of studies, the application of PAM as the conditioner or stabilizer of soil structure may increase the cohesive affinity of grains on the upper soil layer, maintain a good soil structure, avoid soil crust and increase the infiltration rate of $\operatorname{soil}^{4}$ and reduce surface runoff and avoid soil loss 5 . In addition, it can also effectively restrain soil moisture evaporation. Therefore, it has wide application in the improvement of soil infiltration capacity, prevention and control of water and soil erosion ${ }^{6,7}$ and engineering construction ${ }^{8}$. Sodium polyacrylate (PAA) is a type of carboxylized water-soluble macromolecule absorbent resin. It can improve the state of soil particles and lower bulk density and improve soil porosity, impact resilience and water retentivity in soil ${ }^{9}$. Although research on PAA has achieved numerous results, most of these papers have focused on the study on the application methods of PAA ${ }^{10}$, effects of fertilizer maintenance ${ }^{11,12}$, comparison of the effects of PAA on water retention and fertilizer maintenance with those of other water holding materials, synthesis of PAA with other new organic and inorganic water retention pharmaceutics ${ }^{13}$ and properties of PAA such as water absorption, fertilizer maintenance ${ }^{14}$ and salt absorption ${ }^{15}$. Wote super absorbent is an organicinorganic compound water retention agent synthesized using the hybridizing process of polyacrylic acid and attapulgite. After five repetitions moisture absorption and release, its water absorbing capacity is still greater than $85 \%$ of its original water absorbing capacity ${ }^{16-18}$. Compared with all other existing types of water retention agents, Wote super absorbent can not 
only reduce the cost of raw materials, it can also improve the crop yield by $30 \%$, thus it has broad application prospects.

However, as a new material, studies on the proper amount of application macromolecule absorbent resins in Heilu soil of the Loess Plateau and effects on soil moisture characteristics have yet to achieve unified conclusions ${ }^{19}$. The main characteristics of the Loess Plateau are that it has an annual rainfall of less than $400 \mathrm{~mm}$ and poor water retention, making it prone to severe water and soil erosion. The application of macromolecule absorbent resins can improve soil structure, promote water retaining capacity, reduce ineffective evaporation and improve utilization efficiency of soil moisture, thus improving the water environment of Heilu soil in arid and semi-arid regions. Therefore, in this paper we discuss the effects of different macromolecule absorbent resins on the water holding, retention and furnishing capacity of Heilu soil, by studying the adjustment of water holding capacity and evaporative resistance of soil using super absorbent resins such as PAA, PAM and Wote super absorbent as soil conditioners, in the hope of providing the basis for the practical application of different macromolecule absorbent resins in Heilu soil on the Loess Plateau.

\section{EXPERIMENTAL}

The test materials used are as follows: Wote multi-function super absorbent (synthesized using the hybridizing process of polyacrylic acid and attapulgite), manufactured by Shengli Oilfield Chang'an Holding Group, China; PAM (polyacry lamide), manufactured by SNF Co., France and polyacrylate sodium (PAA), manufactured by Northwestern Polytechnical University, China. The Heilu soil was collected from $0-20 \mathrm{~cm}$ soil on farmland of Changwu County, Shaanxi Province, China and had an organic content of $12.3 \mathrm{~g} / \mathrm{kg}$.

Soil column filling for saturated hydraulic conductivity and water holding capacity of soil: The cutting-ring method was adopted in this experiment. $50 \mathrm{~g}$ of soil, after being screened on a $2 \mathrm{~mm}$ screen, was blended with different macromolecule absorbent resins and this was loaded at the position of $2 / 3$ of the height of a standard cutting ring (with a volume of $100 \mathrm{~cm}^{3}$ ). A layer of soil of the same kind was then placed on top of this until filling to the top of the soil column, with a soil volume weight of $1.32 \mathrm{~g} / \mathrm{cm}^{3}$. The amounts of macromolecule absorbent resins were contrast (CK), $0 \mathrm{mg} / \mathrm{kg}, 0.053 \mathrm{mg} / \mathrm{kg}$ (PAA1, PAM1, W1 (Wote1)), $0.106 \mathrm{mg} / \mathrm{kg}$ (PAA2, PAM2, W2 (Wote2)), $0.159 \mathrm{mg} / \mathrm{kg}$ (PAA3, PAM3, W3 (Wote3)) and 0.212 $\mathrm{mg} / \mathrm{kg}$ (PAA4, PAM4, W4 (Wote4)). The amount of soil used (converted into oven-dried soil) was $132 \mathrm{~g}$.

Soil column filling for soil evaporative resistance experiment: For the evaporative resistance experiment, a soil column with a height of $20 \mathrm{~cm}$ and diameter of $10 \mathrm{~cm}$ was used. The amounts of macromolecule absorbent resins were, respectively as follows: contrast $(\mathrm{CK}), 0 \mathrm{mg} / \mathrm{kg}, 54.5 \mathrm{mg} / \mathrm{kg}$ (PAA1, PAM1, W1 (Wote1)), 112.9 mg/kg (PAA2, PAM2, W2 (Wote2)), $169.4 \mathrm{mg} / \mathrm{kg}$ (PAA3, PAM3, W3 (Wote3)) and
$225.8 \mathrm{mg} / \mathrm{kg}$ (PAA4, PAM4, W4 (Wote4)). The filling method was the same as that used for the loading cutting ring sample. The volume weight of soil loading was $1.24 \mathrm{~g} / \mathrm{cm}^{3}$. The indoor temperature was $25{ }^{\circ} \mathrm{C}$ and the relative humidity was $65 \%$. The amount of soil used (converted into oven-dried soil) was 2070 g. The soil evaporative resistance experiment was conducted under the conditions of constant temperature and humidity, i.e., the soil column was placed into a room of constant temperature and humidity, after being fully saturated (temperature of $25^{\circ} \mathrm{C}$ and relative humidity of $65 \%$ ). The soil column was weighed every two to three days and the soil evaporation capacity of the duration was calculated. The determination began on May 10 and was completed on June 30 .

Experimental determination and method: The saturated hydraulic conductivity of the soil was determined using the hydrostatic head method ${ }^{20}$ and the calculation formula of saturated hydraulic conductivity is as follows:

$$
\mathrm{Ks}=\left(\frac{\mathrm{QL}}{(\mathrm{a} \times \mathrm{t} \times \mathrm{H})}\right)
$$

where Ks is the saturated hydraulic conductivity $(\mathrm{mm} / \mathrm{h})$; Q is the flow rate $\left(\mathrm{mm}^{3}\right) ; \mathrm{L}$ is the length of the soil column, equal to $50 \mathrm{~mm}$ (height of cutting ring); $\mathrm{A}$ is the sectional area of soil column ( $\mathrm{mm}^{2}$, the diameter is the cross-sectional area of a $50 \mathrm{~mm}$ standard cutting ring); $\mathrm{t}$ is the time (h) and $\mathrm{H}$ is the height of the water head ( $\mathrm{mm})$.

The soil moisture characteristic curve was determined using the high speed centrifugal method ${ }^{21}$ and the suction and centrifugal time corresponding to the rotation speed of the centrifugal machine are shown in Table-1. The soil was weighed after each completion of centrifugation. The soil sample was placed in the drying cabinet for drying after the experiment and weighed, the corresponding soil moisture contents under different soil moisture suctions were calculated and the soil moisture characteristic curve was fitted. All curves in the experiment can be fitted into the following power function:

$$
\mathrm{y}=\mathrm{a} \theta \mathrm{x}^{-\mathrm{b}}
$$

where $\theta$ is the mass water content of soil on the soil layer calculated, $x$ is the value of soil suction (MPa) and $a$ and $b$ are the fitting parameters of the equation. According to this fitted equation, the soil moisture contents of each undisturbed soil sample under 0.03 and $1.5 \mathrm{MPa}$ can be calculated, i.e., field water-holding capacity and wilting moisture capacity (humidity).

The specific water capacity of soil can indicate the effectiveness of the water and water furnishing ability of the $\mathrm{soil}^{24}$, the quantity of which can be represented by the equation below:

$$
C_{y}=-\frac{d y}{d x}=a b x^{-(b+1)}
$$

where $\mathrm{C}_{\mathrm{y}}$ is the specific water capacity; $\mathrm{y}$ is the soil moisture content; $\mathrm{x}$ is the soil moisture suction; $\mathrm{a}$ and $\mathrm{b}$ are parameters,

TABLE-1

SPEED AND TIME OF SOIL WATER POTENTIAL CORRESPONDING TO HIGH-SPEED CENTRIFUGE

\begin{tabular}{lccccccccccc}
\hline Water suction $(\mathrm{Mpa})$ & 0.01 & 0.02 & 0.04 & 0.06 & 0.08 & 0.1 & 0.2 & 0.4 & 0.6 & 0.8 & 1.0 \\
\hline Rotation speed $(\times 1000 \mathrm{rpm} / \mathrm{min})$ & 0.98 & 1.39 & 1.96 & 2.40 & 2.77 & 3.10 & 4.39 & 6.20 & 7.60 & 8.77 & 9.60 \\
Time $(\mathrm{min})$ & 26 & 36 & 45 & 51 & 55 & 58 & 68 & 77 & 83 & 87 & 93 \\
\hline
\end{tabular}


among which a value reflects the water retention capability of the soil. $\mathrm{a} \times \mathrm{b}$ value is the soil moisture suction and $\mathrm{x}$ is the specific water capacity when the suction is $1 \mathrm{MPa}$, which reflects the water furnishing ability of soil. The greater the $\mathrm{a} \times$ $\mathrm{b}$ value, the stronger the water furnishing ability of the soil. The corresponding specific water capacity of the soil under different suctions can be calculated using this equation.

Statistical methods: The data obtained in the experiment were the arithmetic mean values of three repeated calculations. The data of variance obtained through analysis was analyzed and processed using statistics and relevant mathematical statistical software (DPS10.0).

\section{RESULTS AND DISCUSSION}

Model of soil moisture characteristic curve and analysis of water retention ability: The soil moisture characteristic curve, an important tool in the study of soil moisture characteristics, can reflect both the condition of moisture retention of soil and the relationship between the quantity and energy of the soil moisture. Therefore, it is also known as the soil moisture retention curve $\mathrm{e}^{21,22}$.

The differences in the effects of the different macromolecule absorbent resins on the improvement of soil contributes to the different physicochemical characteristics of the soil. Therefore, there are differences in their respective water conservation performances, which can be reflected by the differences shown in the soil moisture characteristic curve. Water characteristic curve indicates the water retention ability of soil, i.e. under the same suction, the higher the curve, the stronger the water retention ability and vice versa.

As shown in Table-2, all of the fitted equations of the water characteristic curves in this study reach significant levels $(p<0.01)$. After the application of macromolecule absorbent resins in the soil, the parameter a in its fitted equation clearly increases. This indicates that the application of macromolecule absorbent resins can significantly improve the water retention ability of the soil.

It can be seen from the water characteristic curve that after the application of the three types of macromolecule absorbent resins (Figs. 1 and 2), the water retention ability of the contrast is the lowest. However, the soil moisture characteristic curve after the application of macromolecule absorbent

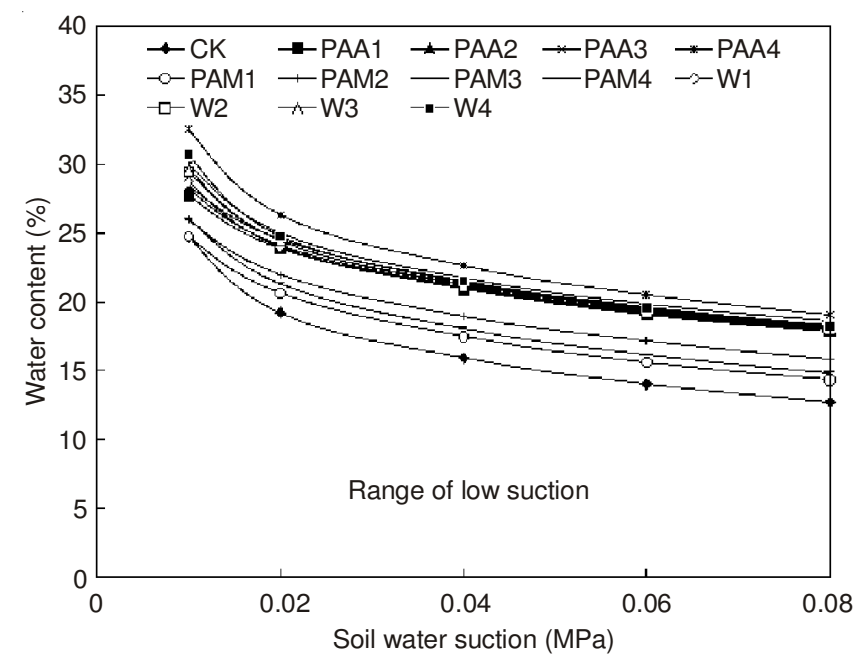

Fig. 1. Comparison of capability of soil water retention among different treatments with macromolecule absorbent resins under range of low suction

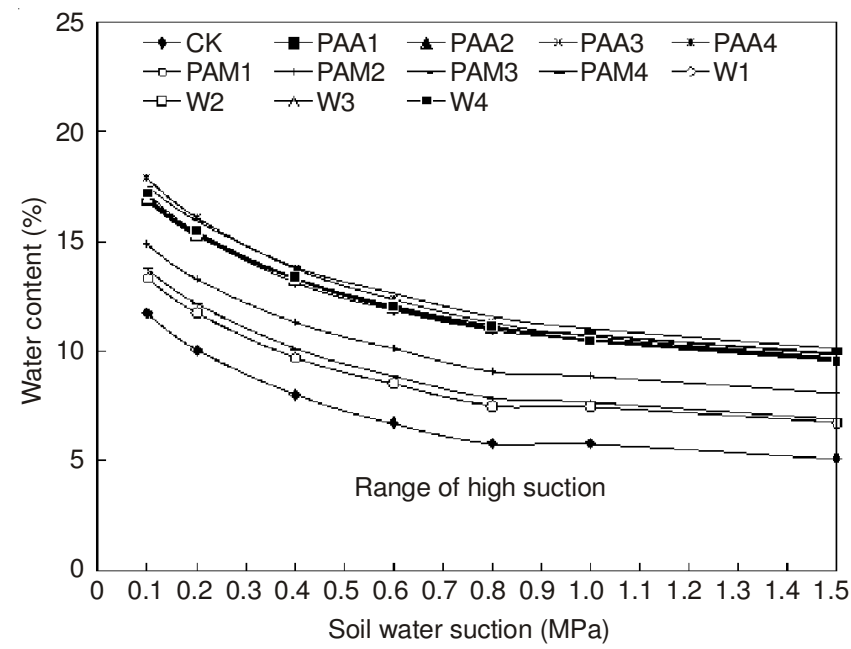

Fig. 2. Comparison of capability of soil water retention among different treatments with macromolecule absorbent resins under range of high suction

resins is apparently higher than that of the contrast, which indicates that it improves the water retention ability of the soil. When the soil moisture suction is $<0.1 \mathrm{MPa}$ (low suction section) (Fig. 1), among which the water retention ability of

\begin{tabular}{|c|c|c|c|c|c|c|}
\hline \multicolumn{7}{|c|}{$\begin{array}{c}\text { TABLE-2 } \\
\text { MATHEMATICAL MODEL AND CORRELATIVE COEFFICIEI }\end{array}$} \\
\hline \multicolumn{2}{|c|}{ Polymer materials } & Mathematical model & $\mathrm{a}$ & $a b$ & $\mathrm{~b}+1$ & $\mathrm{R}^{2}$ \\
\hline \multicolumn{2}{|c|}{$\mathrm{CK}$} & $y=5.79 x^{-0.31}$ & 5.79 & 1.79 & 1.31 & $0.9973 * *$ \\
\hline \multirow{4}{*}{ PAA } & PAA1 & $y=10.77 x^{-0.20}$ & 10.77 & 2.15 & 1.20 & $0.9984 * *$ \\
\hline & PAA2 & $y=10.74 x^{-0.21}$ & 10.74 & 2.26 & 1.21 & $0.9982 * *$ \\
\hline & PAA3 & $y=10.80 x^{-0.21}$ & 10.80 & 2.27 & 1.21 & $0.9983 * *$ \\
\hline & PAA4 & $y=10.71 x^{-0.24}$ & 10.71 & 2.57 & 1.24 & $0.9946 * *$ \\
\hline \multirow{4}{*}{ PAM } & PAM1 & $y=7.50 x^{-0.26}$ & 7.50 & 1.95 & 1.26 & $0.9982 * *$ \\
\hline & PAM2 & $y=8.91 x^{-0.23}$ & 8.91 & 2.05 & 1.23 & $0.9983 * *$ \\
\hline & PAM3 & $y=11.05 x^{-0.21}$ & 11.05 & 2.32 & 1.21 & $0.9953 * *$ \\
\hline & PAM4 & $\mathrm{y}=7.71 \mathrm{x}^{-0.26}$ & 7.71 & 2.00 & 1.26 & $0.9979 * *$ \\
\hline \multirow{4}{*}{ Wote } & W1 & $y=10.59 x^{-0.21}$ & 10.59 & 2.22 & 1.21 & $0.9973 * *$ \\
\hline & W2 & $y=10.52 x^{-0.22}$ & 10.52 & 2.31 & 1.22 & $0.9960 * *$ \\
\hline & W3 & $y=10.56 x^{-0.22}$ & 10.56 & 2.32 & 1.22 & $0.9962 * *$ \\
\hline & W4 & $y=10.50 x^{-0.23}$ & 10.50 & 2.42 & 1.23 & $0.9928 * *$ \\
\hline
\end{tabular}


the PAA4 application is the strongest, followed by that of the PAM3 application. Except for the PAM3 application, all of the water retention abilities of PAM applications are lower than those of the PAA and Wote super absorbent applications. When the soil moisture suction is $0.1-1.5 \mathrm{MPa}$ (Fig. 2), the water retention ability of the PAA4 application is the strongest and that of PAM1 is relatively weak. The water characteristic curves of the Wote super absorbent application of different amounts used almost coincide, which indicates that there is no remarkable difference among its applications.

Effects of different macromolecule absorbent resins on water furnishing ability: It can be observed from Table-1 that, after the application of the macromolecule absorbent resins, the $\mathrm{a} \times \mathrm{b}$ value increases significantly and has a tendency of increasing along with the increase of the amount of macromolecule absorbent resins used. This indicates that the application of macromolecule absorbent resins improves the water furnishing ability of soil and the effect of PAM4 is the best.

The slope of the soil moisture characteristic curve is known as specific water capacity and refers to the amount of water which can be released or drawn from the soil of a unit mass when the unit suction changes. It can also indicate the effectiveness of the soil moisture and water furnishing volume and serves as an important index for the evaluation of drought resistance of the soil ${ }^{23}$. Under the same soil suction, the greater the specific water capacity is, the stronger the water furnishing ability will be.

It can be observed from Table- 3 that, in the section of soil moisture suction of 0.01-0.04, except for the PAA4 application, all of the specific water capacities of the other applications are lower than the capacity of the contrast. In the section of soil moisture suction of $0.04-0.1 \mathrm{MPa}$, the specific water capacities of the applications of PAA4, W2, W3 and W4 are higher than the capacity of the contrast. When the soil moisture suction is greater than $0.1 \mathrm{Mpa}$, the specific water capacities of the soil of different applications are apparently higher than the capacity of the contrast. This indicates that there are certain differences among the effects of macromolecule absorbent resins on the specific water capacity of the soil in the different stages of soil suction. However, the water furnishing ability of the soil can be improved in the sections of moderate and high suctions and when the amount increases to a certain extent, this is beneficial to the improvement of the water furnishing ability of the low suction section of the soil $(<0.1 \mathrm{MPa})$. Among the various applications, the water furnishing ability of the PAA4 application is the strongest.

Effects of different macromolecule absorbent resins on the water transmitting ability of soil: It can be observed from Fig. 3 that, after the application of PAA into the soil, with the increase of its amount, the saturated hydraulic conductivity of the soil decreased significantly and was the lowest in the application of PAA3, which decreased by $46.2 \%$ compared with that of the contrast. This shows that the application of PAA of the amount used in this study decreased the saturated hydraulic conductivity of the soil. A proper amount of PAM and Wote super absorbent improved the saturated hydraulic conductivity of the soil. When the amount used was too large (PAM4 and W4), then the saturated hydraulic conductivity of soil decreased. This shows that suitable amounts of PAM and Wote super absorbent can improve the pores of the soil when mixed with soil, thus promoting water infiltration.

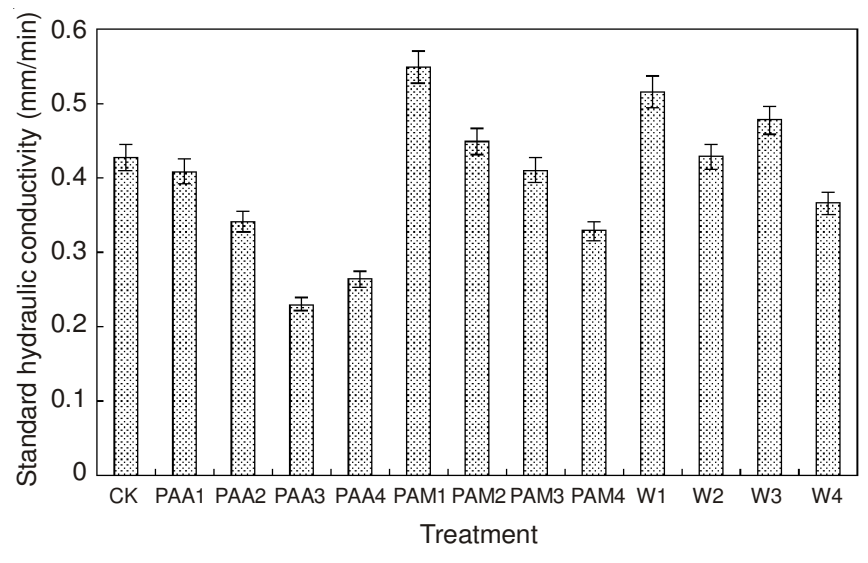

Fig. 3. Effects of different treatments with macromolecule absorbent resins on saturated hydraulic conductivity

Effects of different macromolecule absorbent resins on evaporative capacities of different types of soil: It can be observed from Fig. 4 that the application of macromolecule absorbent resins into soil significantly decreased the evaporation capacity. With the increase of the amount of PAA

\begin{tabular}{|c|c|c|c|c|c|c|c|c|c|c|c|c|}
\hline \multirow{3}{*}{ Treatment } & \multicolumn{11}{|c|}{$\begin{array}{l}\text { TABLE-3 } \\
\text { ATER CAPACITY AMONG DIFFERENT } \\
\text { OLECULE ABSORBENT RESINS (mL/MPa) }\end{array}$} & \\
\hline & \multicolumn{12}{|c|}{ Soil suction (MPa) } \\
\hline & 0.01 & 0.02 & 0.04 & 0.06 & 0.08 & 0.1 & 0.2 & 0.4 & 0.6 & 0.8 & 1 & 1.5 \\
\hline CK & 7.482 & 3.018 & 1.217 & 0.716 & 0.491 & 0.366 & 0.148 & 0.060 & 0.035 & 0.024 & 0.018 & 0.011 \\
\hline PAA1 & 5.411 & 2.355 & 1.025 & 0.630 & 0.446 & 0.341 & 0.149 & 0.065 & 0.040 & 0.028 & 0.022 & 0.013 \\
\hline PAA2 & 5.932 & 2.564 & 1.108 & 0.679 & 0.479 & 0.366 & 0.158 & 0.068 & 0.042 & 0.030 & 0.023 & 0.014 \\
\hline PAA3 & 5.965 & 2.579 & 1.115 & 0.682 & 0.482 & 0.368 & 0.159 & 0.069 & 0.042 & 0.030 & 0.023 & 0.014 \\
\hline PAA4 & 7.762 & 3.286 & 1.391 & 0.842 & 0.589 & 0.447 & 0.189 & 0.080 & 0.048 & 0.034 & 0.026 & 0.016 \\
\hline PAM1 & 6.457 & 2.696 & 1.126 & 0.675 & 0.470 & 0.355 & 0.148 & 0.062 & 0.037 & 0.026 & 0.020 & 0.012 \\
\hline PAM2 & 5.910 & 2.520 & 1.074 & 0.652 & 0.458 & 0.348 & 0.148 & 0.063 & 0.038 & 0.027 & 0.020 & 0.012 \\
\hline PAM3 & 6.104 & 2.638 & 1.140 & 0.698 & 0.493 & 0.376 & 0.163 & 0.070 & 0.043 & 0.030 & 0.023 & 0.014 \\
\hline PAM4 & 6.638 & 2.772 & 1.157 & 0.694 & 0.483 & 0.365 & 0.152 & 0.064 & 0.038 & 0.027 & 0.020 & 0.012 \\
\hline W1 & 5.849 & 2.529 & 1.093 & 0.669 & 0.472 & 0.361 & 0.156 & 0.067 & 0.041 & 0.029 & 0.022 & 0.014 \\
\hline W2 & 6.374 & 2.736 & 1.175 & 0.716 & 0.504 & 0.384 & 0.165 & 0.071 & 0.043 & 0.030 & 0.023 & 0.014 \\
\hline W4 & 6.965 & 2.969 & 1.266 & 0.769 & 0.540 & 0.410 & 0.175 & 0.075 & 0.045 & 0.032 & 0.024 & 0.015 \\
\hline
\end{tabular}




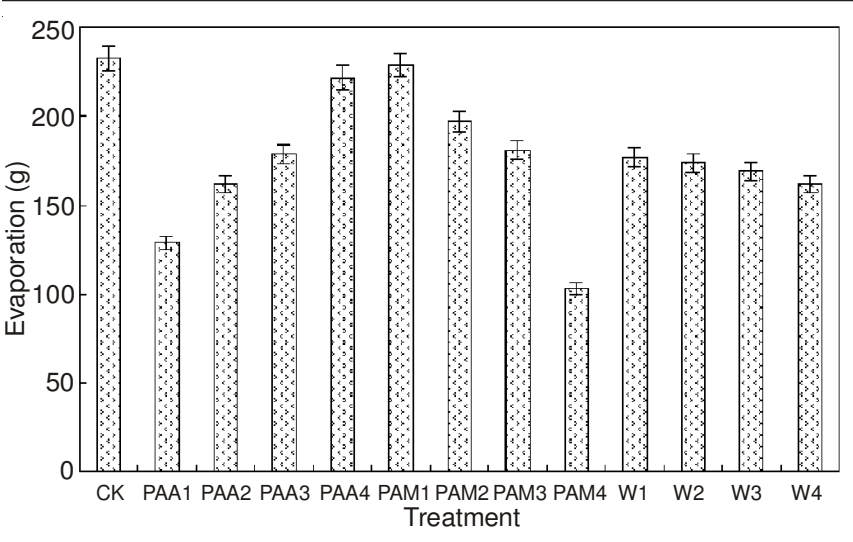

Fig. 4. Effects of different treatments with macromolecule absorbent resins on soil evaporation

used, the soil evaporation capacity had a tendency to first increase then decrease, but was still notably lower than that of the contrast. However, as for PAM and Wote super absorbent, with the increase of amount used, the soil evaporation capacity decreased significantly, especially in that the decreasing amplitude of the soil evaporation capacity of the PAM4 application was the highest, at $44.2 \%$ of that of the contrast. This shows that the application of macromolecule absorbent resins can effectively reduce the soil evaporation capacity and improve soil moisture, but it is important to note the amount used.

Effects of different macromolecule absorbent resins on soil moisture constant: Saturated moisture capacity refers to the maximum moisture capacity when all soil pores are full of moisture. Field moisture capacity refers to the upper limit of available water of plants and can indicate the level of water retention ability of the soil ${ }^{24}$ and wilting moisture capacity refers to the lower limit of soil available water. Available moisture capacity of soil is the difference value between the field moisture capacity and wilting moisture capacity.

It can be observed from Table-4 that the application of macromolecule absorbent resins into the soil considerably improved the moisture constant of the soil. With the increase of macromolecule absorbent resins, the saturated moisture capacity, field moisture capacity and available moisture capacity of the soil all improved. With the increase of the amount of PAM, the wilting water capacity of the soil had a tendency to first increase then decrease and except for contrast, the wilting moisture capacity of PAM4 was the lowest. This shows that the application of macromolecule absorbent resins improved the gross porosity of the soil (saturated moisture capacity) and the capillary porosity (field moisture capacity) also increased correspondingly, thus increasing the available moisture capacity of soil. Among the various applications, the capacity after application of PAA4 was notably higher than those after the other applications. Compared with the contrast,

\begin{tabular}{|c|c|c|c|c|}
\hline \multicolumn{5}{|c|}{$\begin{array}{c}\text { TABLE-4 } \\
\text { EFFECTS OF DIFFERENT MACROMOLECULE ABSORBENT } \\
\text { RESINS ON SOIL MOISTURE CHARACTERISTICS }\end{array}$} \\
\hline Treatment & $\begin{array}{l}\text { Saturated } \\
\text { moisture } \\
\text { capacity } \\
(\%)\end{array}$ & $\begin{array}{c}\text { Field } \\
\text { moisture } \\
\text { capacity } \\
(\%)\end{array}$ & $\begin{array}{c}\text { Available } \\
\text { moisture } \\
\text { capacity } \\
(\%)\end{array}$ & $\begin{array}{c}\text { Wilting } \\
\text { moisture } \\
\text { capacity } \\
(\%)\end{array}$ \\
\hline CK & $36.7 \mathrm{~d}$ & $16.6 \mathrm{~d}$ & $11.5 \mathrm{~d}$ & $5.1 \mathrm{~d}$ \\
\hline PAA1 & $40.8 b$ & $21.3 b$ & $11.3 \mathrm{~d}$ & $9.9 \mathrm{a}$ \\
\hline PAA2 & $41.3 b$ & $21.9 \mathrm{~b}$ & $12.1 \mathrm{c}$ & $9.9 \mathrm{a}$ \\
\hline PAA3 & $41.6 \mathrm{~b}$ & $22.1 \mathrm{~b}$ & $12.1 \mathrm{c}$ & $9.9 \mathrm{a}$ \\
\hline PAA4 & $45.8 \mathrm{a}$ & $24.2 \mathrm{a}$ & $14.5 \mathrm{a}$ & $9.7 \mathrm{a}$ \\
\hline PAM1 & $37.2 \mathrm{c}$ & $18.2 \mathrm{c}$ & $11.4 \mathrm{~d}$ & $6.7 \mathrm{c}$ \\
\hline PAM2 & $38.4 \mathrm{c}$ & $19.5 \mathrm{c}$ & $11.4 \mathrm{~d}$ & $8.1 \mathrm{~b}$ \\
\hline PAM3 & $42.8 \mathrm{~b}$ & $22.6 \mathrm{~b}$ & $12.4 \mathrm{bc}$ & $10.1 \mathrm{a}$ \\
\hline PAM4 & $38.8 \mathrm{c}$ & $18.7 \mathrm{c}$ & $11.7 \mathrm{~cd}$ & $6.9 \mathrm{c}$ \\
\hline W1 & $42.1 \mathrm{~b}$ & $21.6 \mathrm{~b}$ & $11.9 \mathrm{~cd}$ & $9.7 \mathrm{a}$ \\
\hline W2 & $42.7 b$ & $22.2 \mathrm{~b}$ & $12.6 b c$ & $9.6 \mathrm{a}$ \\
\hline W3 & $42.8 \mathrm{~b}$ & $22.3 b$ & $12.7 b c$ & $9.7 \mathrm{a}$ \\
\hline W4 & $44.1 \mathrm{a}$ & $23.0 \mathrm{~b}$ & $13.4 \mathrm{~b}$ & $9.6 \mathrm{a}$ \\
\hline
\end{tabular}

the available moisture capacity increased $26.1 \%$. The second highest was that after the application of W4, which increased by $16.5 \%$ compared with that of the contrast.

Analysis on correlation between water retention ability and water furnishing ability of soil and different moisture parameters: It can be observed from Table-5 that the water retention ability and water furnishing ability of the soil are either significantly or very significantly positively correlated with the saturated moisture capacity, field moisture capacity, available moisture capacity and wilting moisture capacity, but are either significantly or very significantly negatively correlated with water loss speed $(b+1$ value) and slightly negatively correlated with saturated hydraulic conductivity. This shows that it is feasible to promote the improvement of water retention ability and water furnishing ability of soil, by improving the saturated moisture capacity, field moisture capacity, available moisture capacity of soil.

\section{Conclusion}

In this study, we observed that the application of different macromolecule absorbent resins into soil improved its water retention ability and water furnishing ability and promoted the increase of availability of soil water. In addition, it also reduced the evaporation capacity of the soil. As compared with other macromolecule absorbent resins, the effect of the amount of PAM used on the water retention ability of soil was more sensitive. With the increase of the amount of PAM used, the water retention ability of the soil improved significantly. However, among the different macromolecule absorbent resins, the water retention ability of PAA4 was the strongest. In addition to the improvement of the water retention ability of soil due to

\begin{tabular}{|c|c|c|c|c|c|c|}
\hline \multicolumn{7}{|c|}{$\begin{array}{c}\text { TABLE-5 } \\
\text { CORRELATION ANALYSIS OF WATER PARAMETERS }\end{array}$} \\
\hline Factors & $\begin{array}{l}\text { Saturated hydraulic } \\
\text { conductivity }\end{array}$ & $\begin{array}{c}\text { Saturated } \\
\text { moisture capacity }\end{array}$ & $\begin{array}{l}\text { Field moisture } \\
\text { capacity }\end{array}$ & $\begin{array}{c}\text { Available } \\
\text { moisture capacity }\end{array}$ & $\begin{array}{l}\text { Wilting moisture } \\
\text { capacity }\end{array}$ & $b+1$ value \\
\hline a value & -0.2455 & $0.8441 * *$ & $0.9383 * *$ & $0.4861 *$ & $0.9997 * *$ & $-0.9274 * *$ \\
\hline $\mathrm{a} \times \mathrm{b}$ value & -0.3992 & $0.9820 * *$ & $0.9850 * *$ & $0.8528 * *$ & $0.8583 * *$ & $-0.6402 *$ \\
\hline
\end{tabular}


the application of macromolecule absorbent resins, the water furnishing ability was also improved significantly, thus contributing to the improvement of the soils drought resistance. Within the soil suction range of $0.1-1.5 \mathrm{MPa}$, the application of PAA4 improved the water furnishing ability of the soil. The decreasing amplitude of soil evaporation after PAM4 application was the greatest, being $44.2 \%$ of that of the contrast and the evaporation of the PAA application was lower than that of the contrast. In addition, the application of macromolecule absorbent resins slowed the moisture release process of the soil, allowing more moisture to be released, which was beneficial to the absorption and utilization of soil moisture by crops.

The application of macromolecule absorbent resins improved the gross porosity of the soil, thereby improving the saturated moisture capacity of soil and also increased the capillary porosity of the soil to significantly improve the field moisture capacity, thus increasing the available moisture capacity of the soil. Among the various applications, the capacity after application of PAA4 was notably higher than those after the other applications. Compared with the contrast, the available moisture capacity increased by $26.1 \%$. The second highest was that after the application of W4, which increased by $16.5 \%$ compared with that of the contrast. The correlation analysis showed the following: The macromolecule absorbent resins promoted the increase of water retention ability and water furnishing ability of the soil by improving the saturated moisture capacity, field moisture capacity and available moisture capacity of the soil. In the different polymer materials tests, it was shown that treatment with PAA $(0.212$ $\mathrm{mg} / \mathrm{kg}$ ) was better than the other treatments, as recommended by the type and amount of polymer water absorbent resin.

\section{ACKNOWLEDGEMENTS}

This study is supported by the Public Sector (Agriculture) Special funds for Scientific Research Project (201203077), China 863 Project (2013AA102904) and China Science and Technology support program (2013BAD07B07).

\section{REFERENCES}

1. T.M. Wang, Ecological and Environmental Materials, Tianjin University Press, Tianjin, China (2000).

2. L.D. Zhang, Z.B. Li and Z.K. Ren, Fine Chemicals, 2, 12 (1995).

3. X.X. Zou, Super absorbent Polymer, Chemical Industry Press, Beijing, edn 2 (2002).

4. F.L. Santos and R.P. Serralheiro, J. Agric. Eng. Res., 76, 83 (2000).

5. R.D. Lentz and R.E. Sojka, Soil Sci., 158, 233 (1994).

6. W.S. Xia, T.W. Lei and J.G. Liu, Chinese J. Soil Sci., 1, 33 (2002).

7. A.R. Sepaskhah and A.R. Bazrafshan-Jahrom, Biosystems Eng., 4, 93 (2006).

8. T.B. He and H.J. Hu, Functional Polymers and New Technology, Chemical Industry Press, Beijing (2001).

9. J. Yang, Y.R. Kang, C.W. Zhang, L. Zong, L.M. Yang and A.Q. Wang, Chinese J. Soil Sci., 2, 36 (2005).

10. Z.B. Huang, Y.J. Zhu, M.S. Li and G.F. Zhu, J. Shenyang Agric. Univ., 5-6, 35 (2004).

11. J.J. Du, X.A. Wang, X. Feng, W.D. Kong and Z.W. Liao, J. Zhongkai Agrotech. College, 2, 17 (2004).

12. F.Q. Sun and Y.D. Cui, Technol. Develop. Chem. Ind., 6, 33 (2004).

13. R.F. Liu, H.S. Yang, A. Li and A.Q. Wang, Chinese J. Soil Sci., 2, 37 (2006).

14. H.B. Yin and Y.L. Shi, Chinese J. Soil Sci., 2, 37 (2006).

15. X.H. Tang, L.R. Fan, L. Liu and S.Y. Shen, J. China Three Gorges Univ. (Nat. Sci.), 3, 28 (2006).

16. Y.H. Yang, S.W. Zhao, Z.B. Huang, G.S. Bai and N.N. Liu, Ganhan Diqu Nongye Yanjiu, 5, 24 (2006).

17. R.F. Liu, J.P. Zhang and A.Q. Wang, Trans. Chinese Soc. Agric. Eng., 9, 21 (2005).

18. J.P. Zhang, R.F. Liu and A.Q. Wang, Polym. Mater. Sci. Eng., 5, 22 (2006).

19. X.F. Yun, P. Wu and H. Feng, Res. Soil Water Conserv., 1, 9 (2002).

20. W.A. Jary, Soil Physics, Printed in the United States of America, New York (1991).

21. Z.Y. Shi and S.W. Zhao, Acta Agric. Boreali-Occidentalis Sinica, 6, 8 (1999).

22. Z.D. Lei S.X. Yang and S.C. Xie, Soil Water Dynamics, Tsinghua University Press, Beijing, China (1988).

23. Z.X. Chen and R.Z. Wang, Acta Pedolog. Sin., 3, 16 (1979).

24. K.Y. Li, S.F. Han and Y.S. Li, 13 (1991). 